\title{
Relative performance of the two hands in simple and choice reaction time tasks
}

\author{
M. Nisiyama and L.E. Ribeiro-do-Valle
}

Departamento de Fisiologia e Biofísica, Instituto de Ciências Biomédicas, Universidade de São Paulo, São Paulo, SP, Brasil

\begin{abstract}
There is evidence that the left hemisphere is more competent for motor control than the right hemisphere. This study investigated whether this hemispheric asymmetry is expressed in the latency/duration of sequential responses performed by the left and/or right hands. Thirty-two right-handed young adults (16 males, 16 females; $18-25$ years old) were tested in a simple or choice reaction time task. They responded to a left and/or right visual target by moving their left and/or right middle fingers between two keys on each side of the midline. Right hand reaction time did not differ from left hand reaction time. Submovement times were longer for the right hand than the left hand when the response was bilateral. Pause times were shorter for the right hand than the left hand, both when the responses were unilateral or bilateral. Reaction time results indicate that the putatively more efficient response preparation by the left hemisphere motor mechanisms is not expressed behaviorally. Submovement time and pause time results indicate that the putatively more efficient response execution by the left hemisphere motor mechanisms is expressed behaviorally. In the case of the submovements, the less efficient motor control of the left hand would be compensated by a more intense attention to this hand.
\end{abstract}

Key words: Motor lateral asymmetry; Reaction time; Movement time; Response complexity; Unilateral response; Bilateral response

\section{Introduction}

Cortical and subcortical motor areas in the left hemisphere differ both structurally and functionally from those in the right hemisphere, particularly in right-handed individuals, which comprise about $90 \%$ of the population. It was demonstrated that the left central sulcus gray matter volume exceeds that of the right (1); the cortical motor representation in the left hemisphere is larger than that in the right hemisphere (2), and the right corticospinal tract is correspondingly larger (3). It was also demonstrated that the left motor cortices are more excitable than the right motor cortices, as indicated by their lower threshold for activation by transcranial magnetic stimulation (4-6). Moreover, surround inhibition is more efficient in the left primary motor cortex than in the right primary motor cortex (7), and the right, but not the left, primary motor cortex is deactivated during the performance of ipsilateral distal movements (8).

In right-handers, lesions occurring in the left but not right hemisphere lead to longer reaction times and less accurate movement of the ipsilateral hand in a simple aiming task (9). In a task requiring participants to emulate sequences of hand postures varying in complexity and length, these same lesions cause a trend for reaction time increase and a clearly defined slowing of interresponse times and movement time (10). In addition to impairing movement sequencing, lesions involving the left but not right areas (lateral premotor cortex, parietal cortex, thalamus, striatum, and white matter fascicles) were shown to impair response selection in a variety of tasks (11). These findings have been interpreted as indicating that the left hemisphere motor mechanisms of right-handers play a dominant role in response selection (11), and in the programming and initial execution of responses with sequencing requirements $(9,10)$

A point of interest is to what extent this hemispheric asymmetry of the motor mechanisms is translated into a difference of performance of the two upper limbs. Each hemisphere is mainly involved with the control of movements of the contralateral upper limb, particularly in the case of its distal parts. This is suggested by the predominantly contralateral projections of cortical motor areas to the spinal cord (12), and is clearly highlighted by the deficits demonstrated by patients

Correspondence: L.E. Ribeiro-do-Valle, Departamento de Fisiologia e Biofísica, ICB, USP, Av. Prof. Lineu Prestes, 1524, 05508-000 São Paulo, SP, Brasil. E-mail: ribeiro@icb.usp.br

Received February 25, 2013. Accepted August 28, 2013. First published online December 12, 2013. 
with lesions of the motor areas of the left and the right hemispheres (13). According to Nirkko et al. (8), the primary motor area of each hemisphere controls the movements of the proximal and distal parts of the contralateral upper limb and, to a minor extent, those of the proximal parts of the ipsilateral upper limb. The secondary motor areas of each hemisphere control the movements of the proximal and distal parts of the contralateral upper limb and, to a lesser extent, those of the ipsilateral upper limb. It can be presumed, then, that the right upper limb, especially its distal parts, would commonly demonstrate a superior motor performance compared to the left upper limb.

The widespread preference in the population for using the right hand to perform more difficult daily tasks might be a reflection of its natural superior motor ability. The right hand demonstrates a stronger grip (14), greater movement speed $(5,15-17)$, as evaluated with a tapping task, and greater dexterity (5), as evaluated with a pegboard task. These findings can also be taken as evidence that the left hemispheric specialization for motor control has associated behavioral consequences. The hypothesis of a cause and effect relationship between left hemisphere motor dominance and the better performance of the right hand is reinforced by the fact that the central sulcus gray matter volume is positively correlated with performance asymmetry on maximum tapping rate (1), the fact that the size of the primary motor cortex is significantly correlated with performance asymmetry in tasks requiring wrist or finger movements (2), and the fact that the lateral asymmetry of primary motor cortex excitability is strongly correlated with asymmetries of manual movement speed and dexterity (5).

Interestingly, in tasks requiring fast responses to a target, contradictory results have been obtained with respect to the relative performance of the two hands. Taking into account the dominance of the left hemisphere for motor control in right-handed individuals, it would be expected that these individuals would typically exhibit a better performance with their right than their left hand. This, however, has not been consistently observed when considering reaction time, which mainly reflects preparatory motor processes [selection and programming processes, according to Klapp (18)], or movement time, which mainly reflects the executive motor process (10). The literature provides evidence of superior performance by the right hand, but there is also sufficient evidence indicating that there are no differences between the two hands or that there is even superior performance by the left hand.

Reaction time was evaluated in several studies. Faster key pressing by the right hand than by the left hand in response to ipsilateral visual stimuli was reported by Tanigushi et al. (19) and Shen and Franz (20) using a simple reaction time task, and by Kerr et al. (21), Rabbitt
(22), and Tanigushi et al. (19) using a choice reaction time task. Of particular interest, the same result was obtained for bilateral key-pressing responses in a simple task (20) and a go/no-go task (23).

In contrast with these findings, faster responses (index and middle finger flexion) by the left hand were reported by Annett and Annett (24) in the majority of their righthanded participants in both a simple and a two-choice reaction time task. Ortiz et al. (25) and Goodin et al. (26) described faster unilateral and bilateral electromyographic responses of the extensor digitorum with the left hand when compared with the right hand in a simple reaction time task. Shorter finger-lifting reaction times to an ipsilateral target stimulus for the left hand were also described by Barthelemy and Boulinguez (27). In a subsequent study, in which the responding hand was either cued (validly or invalidly) or not cued, the authors verified the same left hand advantage for the invalid and neutral conditions (28).

More often, however, the two hands were observed reacting with the same latency. This result was reported by Rabbitt (22) and Di Stefano et al. (29) for a keypressing response in a simple task, by Anson and Bird (30) for a finger-extension response in a simple task, by Semjen and Gottsdanker (31) for an eight-taps finger response in a choice task, and by Schröter and Leuthold (32) for a single key-pressing response and a three keys consecutive pressing response in a choice task. It was also described for a bilateral key-pressing response by Kerr et al. (21) and Di Stefano et al. (29), for a bilateral finger extension response by Anson and Bird (30), and for a bilateral five-taps finger response by Glencross et al. (33), all in simple tasks.

Movement time was evaluated less often than reaction time. Schröter and Leuthold (32) found shorter movement times by the right hand when right-handers responded to a visual target by either pressing a single key with the index finger or sequentially pressing three keys with the index, ring, and middle fingers. Balfour et al. (34) reported similar movement times for the two sides in a task that required one, two, four, or eight repetitions of unilateral sequential tapping by the index and middle fingers. Semjen and Gottsdanker (31) observed that the right hand was faster than the left at performing an eight-taps finger response in which the second or the third tap had to be accented, but not when performing the same response accenting the first, fifth, or sixth tap.

It is not clear why the expected superior performance of the right hand of right-handed individuals in reaction time tasks was not observed in several studies. In those studies showing similar lateral performance, perhaps the required responses were not complex enough to reveal the left hemisphere dominance for motor control. The right hemisphere motor mechanisms might be as efficient as the left hemisphere motor mechanisms in controlling the preparation and execution 
of this kind of response. It is also possible that the use of simple reaction time tasks to test the participants in some of these studies have allowed advanced partial preparation of the response by the right hemisphere motor mechanisms. These factors may have minimized the disadvantage of these mechanisms. Finally, there is the possibility that, in addition to the right hemisphere motor mechanisms, the left hemisphere motor mechanisms were mobilized to control the left hand in those studies that evaluated more complex responses (34). In this case the lower efficiency of the right hemisphere motor mechanisms, which controls this hand more directly, would be masked.

In the present study, we investigated whether more consistent lateral differences in reaction time and movement time favoring the right hand can be obtained in choice reaction time tasks than in simple reaction time tasks, and when more complex responses (rather than less complex responses) are required. Since the two hemispheres might prepare the response independently when a bilateral target stimulus is presented and a bilateral response is required $(16,35)$, the influence of the mode of presentation of the stimulus and emitting the response, whether unilaterally or bilaterally, was also evaluated. It is important to note that the stimulusresponse bilateral condition permits a within-trial comparison of the performance of the two sides, practically eliminating several sources of error variability (e.g., fluctuations in arousal or attention level) (36). This should further favor the identification of a lateral difference in reaction time, favoring the right hand.

Both reaction time, assumed to mainly reflect the efficiency of motor preparation $(18,37)$, and movement time, assumed to mainly reflect the efficiency of motor execution $(18,37)$, were evaluated. Thus, a behavioral manifestation of the hemispheric asymmetry of these two processes might be revealed. Additionally, the consistency of the behavioral expression of the motor preparation hemispheric asymmetry could be qualitatively contrasted with that of the motor execution hemispheric asymmetry in the same individuals. This contrast was not possible in most of the previous studies, due to the fact that only reaction time was evaluated.

The testing of conditions involving less or more motor preparation, less or more motor organization, and less or more hemispheric independency, and the evaluation of both the efficiency of motor preparation and the efficiency of motor execution in the same individuals distinguish the current study from previous studies in the area. It might evidence a robust faster response preparation and a robust faster response execution by the right hand in reaction time tasks, and indicate the extent that these lateral asymmetries depend on a more complete motor preparation, a more elaborate motor organization, and/or a more independent motor functioning of the two hemispheres.

\section{Material and Methods}

\section{Participants}

Thirty-two undergraduate students (16 males, 16 females) were tested. Their age ranged from 18 to 25 years old. All participants were right-handed according to the Edinburgh Inventory, showed a laterality index of $0.77 \pm 0.13$ (mean $\pm S D$ ), and had normal or corrected-tonormal vision. All were naive concerning the purpose of the experiment.

Written informed consent was obtained from all participants of this study, which was approved by the Ethics Committee of the Instituto de Ciências Biomédicas, Universidade de São Paulo.

\section{Apparatus}

Participants were individually tested in a dimly illuminated and sound-attenuated room. They remained seated at a table with their head positioned in a chin-andfront rest so that their eyes remained $57 \mathrm{~cm}$ away from the screen of a 14-inch video monitor. The background color of this screen was black. A small white spot at its center served as a fixation point (Figure 1, panel A). Two dark red-filled circles ( 0.50 degree of visual angle in diameter) located 2 degrees to the left and right of this fixation point served as placeholders. A change of the color of these circles to light red served to trigger the response of the participant. The presentation of this stimulus was controlled by a computer and programs developed in the MEL2 Professional (Psychology Software Tools, USA) environment.

There were two pairs of optic-switch keys on the table (Figure 1, panel B). The center of each pair was $22 \mathrm{~cm}$ to the right or left of the participant's midsagittal plane. The distance between the center of the keys of each pair was $3.8 \mathrm{~cm}$. The keys were connected to a custom-made coupler unit.

Stimuli-related signals from the parallel port of the computer and response-related signals from the coupler unit were fed into a 1401 plus analog-to-digital converter (Cambridge Electronic Design, UK) set to sample at a rate of $2000 \mathrm{~Hz}$ per channel and stored in a second computer. The Spike 2 software (Cambridge Electronic Design) was used to acquire data, and script programs were used to calculate the latency of the response and the duration of its components (see below) in each trial.

\section{Procedure}

A group of 16 participants (8 males) performed a simple task and another group of 16 participants (8 males) performed a choice task.

Both groups performed four testing sessions, with an interval of four to six weeks between them so as to minimize carryover learning effects. In one of these sessions, the participants responded to the target stimulus by releasing the outer key, pressing the inner key, releasing this key, and 


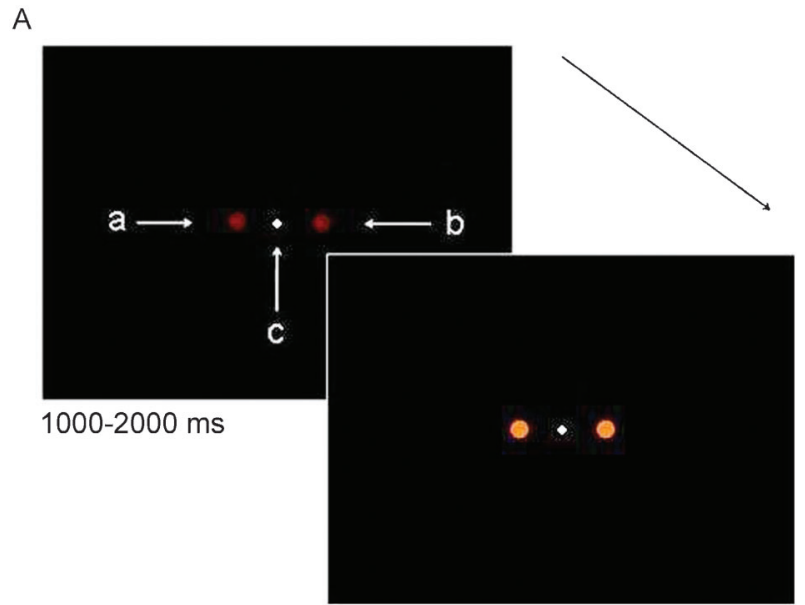

$100 \mathrm{~ms}$

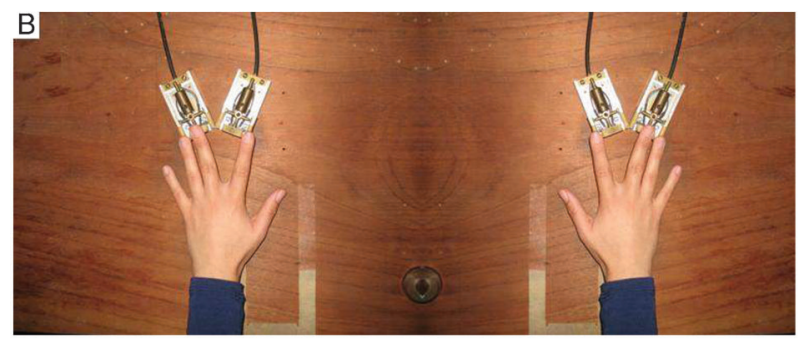

Figure 1. Panel $A$ shows a schematic representation of the sequence of events in a trial. The target stimulus, represented by a left, right or left-right light red filled circle, appeared 1000 to 2000 ms after the beginning of the trial and lasted for 100 ms. "a" indicates the left dark red filled circle. "b" indicates the right dark red filled circle. "c" indicates the central white fixation point. Panel $B$ shows a picture of the responding keys on the left and right sides. Note the initial position of the left and right middle fingers pressing, respectively, the left and right outer keys.

pressing the outer key again (2 submovements response). In another session, the participants responded by repeating this sequence two times (4 submovements response); in another session, by repeating this sequence three times ( 6 submovements response); and in one additional session, by repeating this sequence four times ( 8 submovements response). Each one of these sessions was composed of three blocks of 21 trials.

The participants who performed the simple task were instructed to respond with their left middle finger in one block, with their right middle finger in another block, and with their left and right middle fingers in another block. In each one of these blocks, the target stimulus appeared in the left hemifield in seven trials, in the right hemifield in seven trials, and in both hemifields simultaneously in seven trials (Table 1).

The participants who performed the choice task were instructed to respond in each of the three blocks with their left middle finger during the seven trials in which the target stimulus appeared in the left hemifield, with their right
Table 1. Experimental conditions

\begin{tabular}{ll}
\hline Simple reaction time task & Choice reaction time task \\
\hline Session $(2,4,6$, or & Session $(2,4,6$, or \\
8 submovements) & 8 submovements) \\
Block & Block \\
7 Trials (LT-LH) & 7 Trials (LT-LH) \\
7 Trials (RT-LH) & 7 Trials (RT-RH) \\
7 Trials (LRT-LH) & 7 Trials (LRT-LRH) \\
Block & Block \\
7 Trials (LT-RH) & 7 Trials (LT-LH) \\
7 Trials (RT-RH) & 7 Trials (RT-RH) \\
7 Trials (LRT-RH) & 7 Trials (LRT-LRH) \\
Block & Block \\
7 Trials (LT-LRH) & 7 Trials (LT-LH) \\
7 Trials (RT-LRH) & 7 Trials (RT-RH) \\
7 Trials (LRT-LRH) & 7 Trials (LRT-LRH) \\
\hline
\end{tabular}

A group of 16 participants (8 males) performed four sessions of the simple reaction time task and the other group of 16 participants (8 males) performed four sessions of the choice reaction time task. Each of these sessions consisted of three blocks and each of these blocks consisted of 21 trials. In the simple reaction time task, the left hand was used in a block, the right hand in another block, and the left-right hands in still another block. In the choice reaction time task, the left hand, the right hand, and the left-right hands were used in the same block. LT: left target; RT: right target; LRT: left-right target; LH: left hand; $\mathrm{RH}$ : right hand; $\mathrm{LRH}$ : left-right hands.

middle finger during the seven trials in which the target stimulus appeared in the right hemifield, and with their left and right middle fingers during the seven trials in which the target stimulus appeared in both hemifields simultaneously (Table 1 ).

Before each testing session, the participants performed about 10 practice trials. Prior to each block of trials, each participant was reminded to keep his/her eyes on the fixation point and to respond as fast and as accurately as possible to the target stimulus with their left, right, or left and right hands as appropriate.

Each trial lasted 6.6 to $8.6 \mathrm{~s}$. The target stimulus appeared 1000 to $2000 \mathrm{~ms}$ after the beginning of the trial for a duration of $100 \mathrm{~ms}$. The time window for completing the entire response was 5000 ms.

Responses emitted before the appearance of the target stimulus or less than 150 ms after its onset were considered an anticipation error. Responses emitted more than $2000 \mathrm{~ms}$ after the onset of the target stimulus were considered an omission error. Both criteria were based on previous findings of this laboratory. Other types of errors that could occur in a trial were lack or excess of submovements.

The testing order of the 2, 4, 6, and 8 submovements sessions was counterbalanced among the participants. The testing order of the left, right, and left-right hand blocks was counterbalanced among the participants who performed the simple task. The order of the left, right, and 


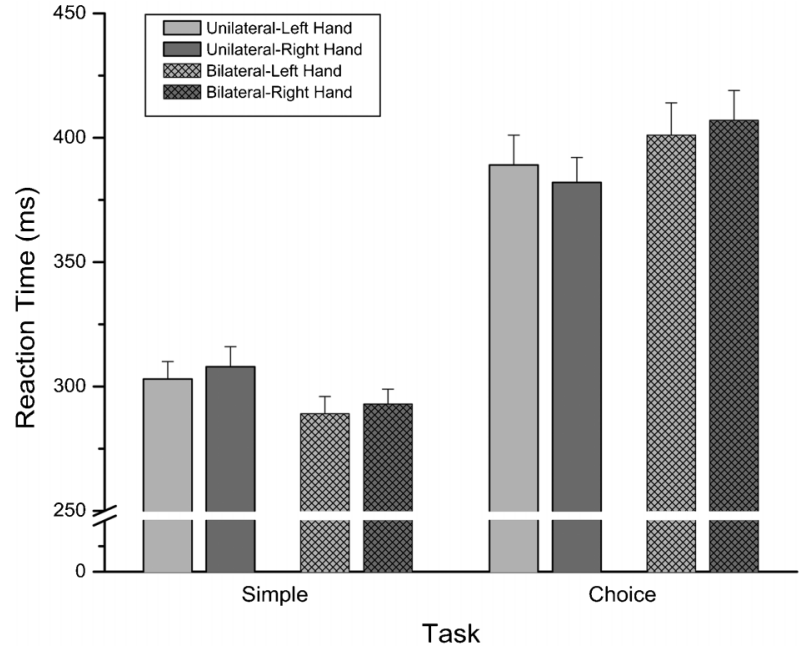

Figure 2. Left hand and right hand reaction times in milliseconds for the unilateral and bilateral responses in the simple and choice tasks. Data are reported as means \pm SE for 16 participants. $\mathrm{P}>0.05$ comparing left and right hands (repeated measures analysis of variance followed by the Tukey test).

left-right target stimulus trials was pseudorandomized; it was the same for all participants.

\section{Data analysis}

Reaction time (interval between flashing of the target and lifting of the finger from the outer key on the same side), submovement times (interval between releasing a key and pressing the other key of the pair), and pause times (interval between pressing and releasing a given key) in each trial were calculated using Spike 2 script language programs. Submovement times were averaged within a trial. Pause times were also averaged within a trial. The median reaction time, submovement time, and pause time of the left and right hands of each participant were calculated for the unilateral and bilateral responses, the 2, 4, 6, and 8 submovements responses, and the simple and choice tasks.

Reaction time, submovement time (mean duration of the response submovements), pause time (mean duration of the response pauses), and movement time (mean duration of the response) data were submitted to mixed repeated measures analyses of variance (ANOVA), with task (simple or choice) as between-subject factor and complexity of the response $(2,4,6$, or 8 submovements), responding mode (unilateral or bilateral), and responding hand (left or right) as within-subject factors. When appropriate, the data were further analyzed by the Tukey test. A significance level of 0.05 was adopted for these analyses.

\section{Results}

The results obtained with each parameter (reaction time, submovement time, pause time, and movement time) are described separately. Nonsignificant main effects and interactions are not reported.

\section{Reaction time}

Figure 2 reports mean reaction times of the left and right hands, which were obtained for the unilateral and bilateral responding modes in the simple and choice tasks.

ANOVA showed a main effect of task $(F 1,30=55.07$; $\left.\mathrm{P}<0.001 ; \eta_{p}^{2}=0.65\right)$ and response complexity $\left(F 3,90=9.04 ; P<0.001 ; \eta_{p}^{2}=0.23\right)$. Reaction time was longer for the choice task than for the simple task. Reaction time was longer for the 6 submovements response than for the 2 submovements response $(P=0.001)$, and for the 8 submovements response than for the 2 and 4 submovements responses $(P<0.001$ and $\mathrm{P}=0.024$, respectively).

Additionally, ANOVA showed an interaction between task and responding mode ( $\mathrm{F} 1,30=43.91 ; \mathrm{P}<0.001$; $\left.\eta_{p}^{2}=0.59\right)$ and between task, responding mode, and responding hand $\left(F 1,30=5.69 ; P=0.024 ; \eta_{p}^{2}=0.16\right)$. The post hoc analysis qualified this triple interaction. It demonstrated that, for the simple task, the left hand and the right hand reaction times were shorter for the bilateral mode than for the unilateral mode $(P=0.004$ and $P=0.001$, respectively) and that for the choice task the left hand and the right hand reaction times were longer for the bilateral mode than for the unilateral mode $(P=0.016$ and $\mathrm{P}<0.001$, respectively).

\section{Submovement time}

Figure 3 shows mean submovement times of the left and right hands that were obtained for the 2, 4, 6, and 8 submovements responses in the unilateral and bilateral responding modes.

ANOVA showed a main effect of response complexity ( $\left.F 3,90=3.64 ; \quad P=0.016 ; \eta^{2}=0.11\right)$ and responding mode $\left(F 1,30=22.17 ; \quad P<0.001 ; \quad \eta_{p}^{2}=0.43\right)$. Submovement time was longer for the 8 submovements response than for the 2 submovements response $(P=0.010)$ and for the bilateral mode than for the unilateral mode.

Additionally, ANOVA showed an interaction between responding mode and responding hand $(F 1,30=21.83$; $\left.\mathrm{P}<0.001 ; \eta_{p}^{2}=0.42\right)$ and between response complexity, responding mode, and responding hand $(F 3,90=2.84$; $\left.\mathrm{P}=0.043 ; \eta_{p}^{2}=0.09\right)$. A post hoc analysis qualified this triple interaction. It demonstrated that the right hand submovement time for the bilateral mode was longer than the left hand submovement time for this same mode for the 4,6 , and 8 submovements responses $(P<0.001)$, and the right hand submovement time for the bilateral mode was also longer than the left and right hand submovement times for the unilateral mode $(P<0.001$ and $P=0.001$, respectively, for the 2 submovements response, and 


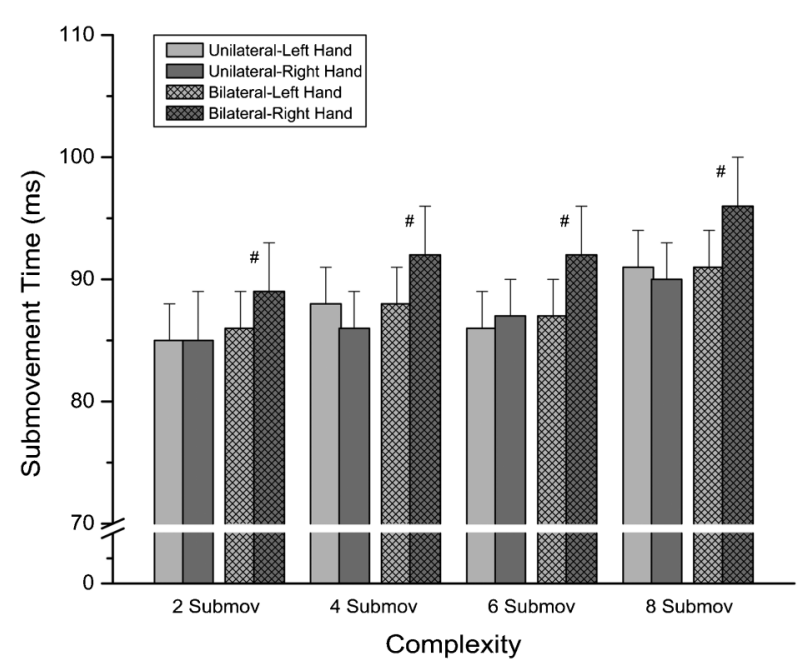

Figure 3. Left hand and right hand submovement times in milliseconds for the unilateral and bilateral responses for the four complexity levels. Data are reported as means \pm SE for 16 participants. Submov: submovements. ${ }^{\#} \mathrm{P}<0.05$ comparing left and right hands (repeated measures analysis of variance followed by the Tukey test).

$\mathrm{P}<0.001$ for the two comparisons, in the case of the 4,6 , and 8 submovements responses).

\section{Pause times}

Figure 4 shows mean pause times of the left and right hands that were obtained for the 2, 4, 6, and 8 submovements responses in the simple and choice tasks (panel A) and in the unilateral and bilateral responding modes (panel B).

ANOVA showed a main effect of task $(F 1,30=6.76$; $\left.P=0.014 ; \eta_{p}^{2}=0.18\right)$, response complexity $(F 3,90=3.51$; $\left.\mathrm{P}=0.019 ; \eta_{\mathrm{p}}^{2}=0.11\right)$, and responding hand $\left(F 1,30=35.93 ; P<0.001 ; \eta_{p}^{2}=0.55\right)$. Pause times were longer for the choice task than for the simple task; they were longer for the 8 submovements response than for the 2 submovements response $(P=0.019)$; and they were longer for the left hand than for the right hand.

ANOVA also showed an interaction between task, response complexity, and responding hand $(F 3,90=6.84$; $\left.\mathrm{P}<0.001 ; \eta_{p}^{2}=0.19\right)$. A post hoc analysis qualified this interaction. It demonstrated that, for the simple task, the right hand pause time was longer for the 4,6 , and 8 submovements responses than for the 2 submovements response $(P=0.026, P<0.001$, and $P<0.001$, respectively), and that, for the choice task, the left hand pause time was longer for the 6 and 8 submovements responses than for the 2 submovements response $(P<0.001)$ and the 4 submovements response $(P=0.021$ and $P<0.001$, respectively). It further demonstrated that, for the simple task and the four complexity levels, the right hand pause time was shorter than the left hand pause time $(P<0.001)$,
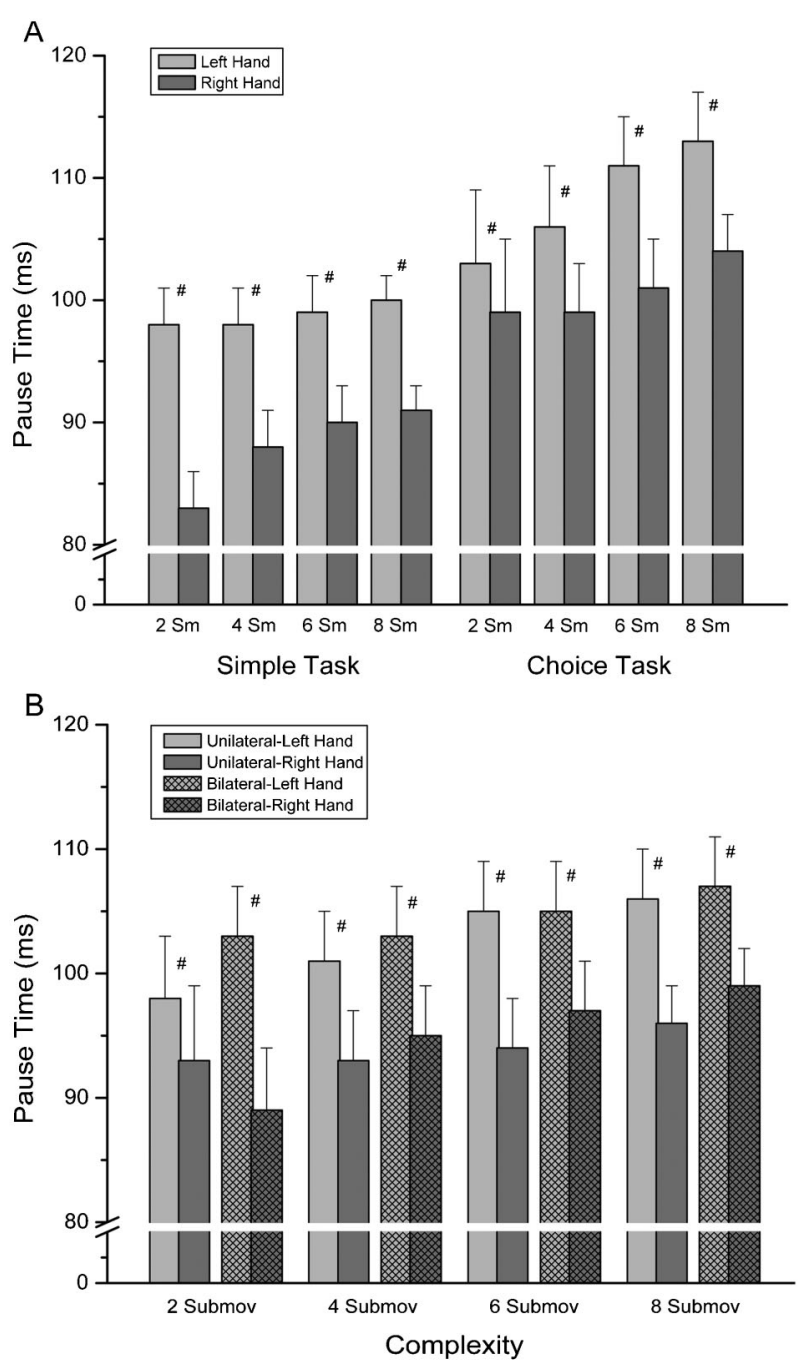

Figure 4. Left hand and right hand pause times in milliseconds for the four complexity levels in the simple and choice tasks (panel $A$ ) and the unilateral and bilateral responses for the four complexity levels (panel $B$ ). Data are reported as means \pm SE for 16 participants. Sm/Submov: submovements. ${ }^{\#} \mathrm{P}<0.05$ comparing left and right hands (repeated measures analysis of variance followed by the Tukey test).

and, for the choice task and the 4, 6 , and 8 submovements responses, the right hand pause time was shorter than the left hand pause time $(P=0.001, P<0.001$, and $P<0.001$, respectively). Figure $4 \mathrm{~A}$ illustrates these results.

ANOVA further showed an interaction between response complexity, responding mode, and responding hand $\left(\mathrm{F} 3,90=16.59 ; \mathrm{P}<0.001 ; \eta_{\mathrm{p}}^{2}=0.36\right)$. A post hoc analysis qualified this interaction. It demonstrated that, for the 2 submovements response, the left hand pause time was longer for the bilateral mode than for the unilateral mode $(P<0.001)$ and the right hand pause time was shorter for the bilateral mode than for the unilateral mode 
$(P=0.029)$. It further demonstrated that, across the four complexity levels and both responding modes, the right hand pause time was shorter than the left hand pause time $(\mathrm{P}<0.001)$. Figure 4B illustrates these results.

\section{Movement times}

ANOVA showed a main effect of response complexity $\left(F 3,90=5.07 ; P=0.003 ; \eta_{p}^{2}=0.15\right)$, responding mode $\left(\mathrm{F} 1,30=21.03 ; \mathrm{P}<0.001 ; \eta_{p}^{2}=0.41\right)$, and responding hand $\left(F 1,30=50.76 ; P<0.001 ; \eta_{p}^{2}=0.63\right)$. Movement times were longer for the choice task than for the simple task, the bilateral mode than the unilateral mode, and the left hand than the right hand. Movement times were longer for the 8 submovements response than for the 2 submovements response $(P<0.001)$.

ANOVA showed an interaction between task, response complexity, and responding hand $(F 3,90=4.19 ; \mathrm{P}=0.008$; $\left.\eta_{p}^{2}=0.12\right)$, and between response complexity, responding mode, and responding hand $(\mathrm{F} 3,90=21.87 ; \mathrm{P}<0.001$; $\eta_{\mathrm{p}}^{2}=0.42$ ).

A post hoc analysis qualified the first interaction. It demonstrated that, for the simple task, the right hand movement time was shorter than the left hand movement time for the 2, 4, 6, and 8 submovements responses $(P<0.001, P<0.001, P=0.001$, and $P<0.001$, respectively) and that, for the choice task, the right hand movement time was shorter than the left hand movement time for the 4, 6, and 8 submovements responses $(P<0.001)$.

Another post hoc analysis qualified the second interaction. It demonstrated that, for the 2 and 4 submovements responses, the right hand movement time was shorter than the left hand movement time for both the unilateral mode $(P<0.001)$ and bilateral mode $(P<0.001$ and $P=0.014$, respectively) and that, for the 6 and 8 submovements responses, the right hand movement time was shorter than the left hand movement time for the unilateral mode $(P<0.001)$.

\section{Accuracy}

Errors were rare for both the simple task $(3.1 \%$ of the trials) and the choice task (3.6\% of the trials), and were represented in the majority of the cases by responses with lack or excess of submovements.

\section{Discussion}

This study was conducted to investigate whether the dominance of the left hemisphere for motor control in right-handed individuals, indicated by anatomical $(1,2)$, physiological (4-8), and clinical (9-11) evidence, is matched by consistent faster right hand responses in reaction time tasks. Responses with 2, 4, 6, and 8 submovements, performed unilaterally or bilaterally, were tested in a simple and a choice task. Reaction time, submovement times, and pause times were all evaluated.
It was demonstrated that the right hand reaction time did not differ from the left hand reaction time in the unilateral and the bilateral responding modes for the simple and choice tasks. It was also shown that the right hand submovement time was longer than the left hand submovement time in the bilateral responding mode for the 4,6 , and 8 submovements responses. Finally, it was shown that the right hand pause time was shorter than the left hand pause time in both responding modes for the four levels of complexity of the response for the simple task, and in both responding modes for the 4, 6, and 8 submovements responses for the choice task. The fact that the same results were obtained in most, if not all, testing sessions (one for each complexity level), separated by about a 1-month interval, indicates that our findings are reliable. This unequivocally distinguishes the present findings from previously reported ones whose robustness was not demonstrated.

The observation that reaction time of the right hand did not differ from reaction time of the left hand in any of our conditions is in agreement with previous research (29-33). It demonstrates that the left hemisphere dominance for motor control is not translated into a superior performance (a shorter latency for responding) of the right hand in reaction time tasks, independently of the complexity of the required response, the responding mode, and of the possibility or not of advanced preparation of the response.

It can be questioned whether this negative result was not related to the particular tasks and/or conditions we used (which might not be the most appropriate), considering that a difference in reaction time between the two hands was reported in several other studies (19,21-27). We cannot exclude these possibilities. We considered more likely, however, that the lateral difference reported in those studies was due to a chance factor (e.g., random neural noise) or to some experimental artifact (e.g., lateral differences in the brightness of the monitor screen or lateral mechanical differences in the response keys).

The most likely explanation for our negative finding is that there occurs an interaction of the associative motor areas of the left hemisphere with those of the right hemisphere during the response programming period, even in the case of the bilateral responding mode. The relatively long time taken by the programming process certainly favors this interaction. The bilateral activity of the associative motor cortex observed during the performance of unilateral hand responses (38) indicates that this interaction is probably the rule. A facilitation of the associative motor mechanisms in the right hemisphere by the associative motor mechanisms in the left hemisphere would increase its programming efficiency and lead to a left hand response latency as short as that of the right hand. In addition to this influence, the associative motor mechanisms in the left hemisphere may directly facilitate the right primary motor cortex, decreasing its activation time and contributing to reduce the response latency of 
the left hand to the level of the right hand response latency. These interactions would hinder the higher efficiency of the left hemisphere motor mechanisms than the right hemisphere motor mechanisms in controlling the preparation of responses to be expressed in behavior.

The longer submovement time of the right hand than the left hand observed in the bilateral responding mode in the case of the 4, 6 , and 8 submovements responses for both tasks could be seen as at odds with the putative higher motor competency of the left hemisphere. It could be supposed that the longer submovement time of the right hand aimed at partially compensating for the shorter pause time of this hand, so as to maintain the two hands relatively synchronized along the trial. This possibility is considerably weakened by the demonstration that the movement time (which includes both submovement time and pause time) of the right hand was shorter (or tended to be shorter) than that of the left hand in all conditions. Another possibility is the occurrence of a selective orienting of attention to the less capable left hand to warrant its proper performance. The fact that the right hand submovement time increased from the unilateral responding mode to the bilateral responding mode while the left hand submovement time did not change is congruent with this idea. The finding of Baldauf and Deubel (39), that attention is biased to the sensory control of more difficult movements, is also in accordance with it.

The advantage observed for the right hand pause time in both responding modes for all levels of complexity in the case of the simple task and for the 4,6 , and 8 submovements responses in the case of the choice task is in agreement with the view that the left hemisphere mechanisms controlling movement direction reversal act faster than those of the right hemisphere $(5,14)$. Since the reciprocal facilitation and inhibition of antagonist pairs of forelimb muscles, which characterizes this reversal operation, probably demands a relatively elaborate neural control, the finding is in perfect agreement with the higher motor competency of the left hemisphere. Some hint regarding the nature of the relevant difference between the motor execution mechanisms of the two hemispheres was provided by llic et al. (6). These authors demonstrated that significantly less short-interval intracortical inhibition occurs in the dominant primary motor cortex when compared with the nondominant primary motor cortex in right-handed individuals, suggesting that the dominant primary motor cortex displays less inhibitory tone than the nondominant primary motor cortex. Since a reduction in the short-interval intracortical inhibition might be necessary for voluntary muscle contraction, this inhibitory tone difference might

\section{References}

1. Herve PY, Mazoyer B, Crivello F, Perchey G, TzourioMazoyer N. Finger tapping, handedness and grey matter provide some advantage for the ease of performing movement reversal with the dominant hand when compared with the nondominant hand.

The findings that the simple task pause time of the right hand increased with response complexity level, while pause time of the left hand did not change, were unexpected. Moreover, the findings that the choice task pause time of the left hand increased with response complexity level, while pause time of the right hand did not change, were also unanticipated. The original prediction was that the pause times of both hands would increase with the level of complexity of the response and that the increase exhibited by the left hand would be larger than that exhibited by the right hand. The obtained results can be tentatively explained by supposing that an increase of online programming of the response on one side occurs as complexity level rises. Possibly, when the response can be prepared in advance, as occurs in simple tasks, online programming is minimum or does not occur for the 2 submovements right hand response. When the response cannot be prepared in advance, as occurs in choice tasks, extra online programming would be necessary for the left hand more complex responses. Clearly, these new findings deserve to be further investigated.

The laterally different submovement and pause times observed in the bilateral responding mode demonstrate that the mechanisms controlling the execution of the movements of the two hands can act with some independence when simultaneously mobilized. A similar conclusion was presented by Foltys et al. (40), based on their observation that transcranial magnetic stimulation of the motor cortex only affects the reaction time of the contralateral hand, independent of whether the response is unilateral or bilateral.

The main results and conclusions of this study can be stated as follows. The putative left hemisphere dominance for response preparation was not demonstrated in the unilateral and bilateral responding modes, in responses constituted by $2,4,6$, and 8 submovements, or in simple and choice tasks. The putative dominance of the left hemisphere for response execution control was demonstrated in both the simple and choice tasks. The shorter pause time exhibited by the right hand in these tasks might be related to the higher competency levels of the left hemisphere primary motor area in controlling response execution. The lateral difference in submovement time and in pause time in the bilateral responding mode suggests some independence in the actions of the mechanisms controlling the execution of the response by the left hand and the right hand in this responding mode. amount in the Rolando's genu area. Neuroimage 2005; 25 : 1133-1145, doi: 10.1016/j.neuroimage.2004.12.062. 
2. Volkmann J, Schnitzler A, Witte OW, Freund H. Handedness and asymmetry of hand representation in human motor cortex. J Neurophysiol 1998; 79: 2149-2154.

3. Kertesz A, Geschwind N. Patterns of pyramidal decussation and their relationship to handedness. Arch Neurol 1971; 24: 326-332, doi: 10.1001/archneur.1971.00480340058006.

4. Triggs WJ, Calvanio R, Macdonell RA, Cros D, Chiappa KH. Physiological motor asymmetry in human handedness: evidence from transcranial magnetic stimulation. Brain Res 1994; 636: 270-276, doi: 10.1016/0006-8993 (94)91026-X.

5. Triggs WJ, Calvanio R, Levine M. Transcranial magnetic stimulation reveals a hemispheric asymmetry correlate of intermanual differences in motor performance. Neuropsychologia 1997; 35: 1355-1363, doi: 10.1016/S00283932(97)00077-8

6. Ilic TV, Jung P, Ziemann U. Subtle hemispheric asymmetry of motor cortical inhibitory tone. Clin Neurophysiol 2004; 115: 330-340, doi: 10.1016/j.clinph.2003.09.017.

7. Shin HW, Sohn YH, Hallett M. Hemispheric asymmetry of surround inhibition in the human motor system. Clin Neurophysiol 2009; 120: 816-819, doi: 10.1016/j.clinph. 2009.02.004.

8. Nirkko AC, Ozdoba C, Redmond SM, Burki M, Schroth G, Hess CW, et al. Different ipsilateral representations for distal and proximal movements in the sensorimotor cortex: activation and deactivation patterns. Neuroimage 2001; 13: 825-835, doi: 10.1006/nimg.2000.0739.

9. Haaland KY, Harrington DL. Hemispheric control of the initial and corrective components of aiming movements. Neuropsychologia 1989; 27: 961-969, doi: 10.1016/00283932(89)90071-7.

10. Harrington DL, Haaland KY. Hemispheric specialization for motor sequencing: abnormalities in levels of programming. Neuropsychologia 1991; 29: 147-163, doi: 10.1016/00283932(91)90017-3.

11. Rushworth MF, Nixon PD, Wade DT, Renowden S, Passingham RE. The left hemisphere and the selection of learned actions. Neuropsychologia 1998; 36: 11-24, doi: 10.1016/S0028-3932(97)00101-2.

12. Kuypers HGJM. Anatomy of the descending pathways. In: Brookhart JM, Mountcastle VB (Editors), Handbook of physiology, Section 1: The nervous system, Vol. 2. Motor control, Part 1. Bethesda: American Physiological Society; 1981. p 597-666.

13. Nowak DA. The impact of stroke on the performance of grasping: usefulness of kinetic and kinematic motion analysis. Neurosci Biobehav Rev 2008; 32: 1439-1450, doi: 10.1016/j.neubiorev.2008.05.021.

14. Teixeira LA. Categories of manual asymmetry and their variation with advancing age. Cortex 2008; 44: 707-716, doi: 10.1016/j.cortex.2006.10.002.

15. Peters $M$, Durding $B$. Left-handers and right-handers compared on a motor task. J Mot Behav 1979; 11: 103111, doi: 10.1080/00222895.1979.10735178.

16. Todor JI, Kyprie PM, Price HL. Lateral asymmetries in arm, wrist and finger movements. Cortex 1982; 18: 515-523, doi: 10.1016/S0010-9452(82)80050-6.

17. Verstynen T, Diedrichsen J, Albert N, Aparicio P, Ivry RB. Ipsilateral motor cortex activity during unimanual hand movements relates to task complexity. I Neurophysiol
2005; 93: 1209-1222, doi: 10.1152/jn.00720.2004

18. Klapp ST. Motor response programming during simple and choice reaction time: the role of practice. J Exp Psychol: Hum Perc Perform 1995; 21: 1015-1027, doi: 10.1037/00961523.21.5.1015.

19. Taniguchi $\mathrm{Y}$, Burle B, Vidal F, Bonnet M. Deficit in motor cortical activity for simultaneous bimanual responses. Exp Brain Res 2001; 137: 259-268, doi: 10.1007/s002210000661.

20. Shen YC, Franz EA. Hemispheric competition in lefthanders on bimanual reaction time tasks. J Mot Behav 2005; 37: 3-9, doi: 10.3200/JMBR.37.1.3-9.

21. Kerr M, Mingay $R$, Elithor $A$. Cerebral dominance in reaction time responses. $B r J$ Psychol 1963; 54: 325-336, doi: 10.1111/j.2044-8295.1963.tb00887.x.

22. Rabbitt P. Hand dominance, attention, and the choice between responses. Q J Exp Psychol 1978; 30: 407-416, doi: 10.1080/00335557843000016.

23. Miller JO, Franz EA. Dissociation of bimanual responses with the Simon effect: on the nonunitization of bimanual responses. J Mot Behav 2005; 37: 146-156, doi: 10.3200/JMBR. 37.2.146-156.

24. Annett $M$, Annett J. Individual differences in right and left reaction time. Br J Psychol 1979; 70: 393-404, doi: 10.1111/j.20448295.1979.tb01709.x.

25. Ortiz TA, Goodin DS, Aminoff MJ. Neural processing in a three-choice reaction-time task: a study using cerebral evoked-potentials and single-trial analysis in normal humans. J Neurophysiol 1993; 69: 1499-1512.

26. Goodin DS, Aminoff MJ, Ortiz TA, Chequer RS. Response times and handedness in simple reaction-time tasks. Exp Brain Res 1996; 109: 117-126, doi: 10.1007/BF00228632.

27. Barthelemy S, Boulinguez P. Manual reaction time asymmetries in human subjects: the role of movement planning and attention. Neurosci Lett 2001; 315: 41-44, doi: 10.1016/ S0304-3940(01)02313-8.

28. Barthelemy S, Boulinguez P. Orienting visuospatial attention generates manual reaction time asymmetries in target detection and pointing. Behav Brain Res 2002; 133: 109116, doi: 10.1016/S0166-4328(01)00446-6.

29. Di Stefano M, Morelli M, Marzi CA, Berlucchi G. Hemispheric control of unilateral and bilateral movements of proximal and distal parts of the arm as inferred from simple reaction time to lateralized light stimuli in man. Exp Brain Res 1980; 38: 197-204, doi: 10.1007/BF00236741.

30. Anson JG, Bird YN. Neuromotor programming: bilateral and unilateral effects on simple reaction time. Hum Movem Sci 1993; 12: 37-50, doi: 10.1016/0167-9457(93)90035-N.

31. Semjen A, Gottsdanker R. Rapid serial movements: relation between the planning of sequential structure and effector selection. In: Jeannerod M (Editor), Attention and performance XIII: Motor representation and control. Hillsdale: Lawrence Erlbaum; 1990. p 409-427.

32. Schröter $H$, Leuthold $H$. Effects of response sequence length on motor programming: a chronometric analysis. Acta Psychol 2008; 128: 186-196, doi: 10.1016/j.actpsy. 2007.12.006

33. Glencross DJ, Piek JP, Barrett NC. The coordination of bimanual synchronous and alternating tapping sequences. $J$ Motor Behav 1995; 27: 3-15, doi: 10.1080/00222895.1995. 9941695.

34. Balfour CA, Clark CR, Geffen GM. Interhemispheric 
differences in the planning and execution of sequences of skilled finger movements. Neuropsychologia 1991; 29: 889905, doi: 10.1016/0028-3932(91)90054-C.

35. Roy EA, MacKenzie C. Handedness effects in kinesthetic spatial location judgements. Cortex 1978; 14: 250-258, doi: 10.1016/S0010-9452(78)80051-3.

36. Kaluzny $\mathrm{P}$, Palmeri A, Wiesendanger $\mathrm{M}$. The problem of bimanual coupling: a reaction time study of simple unimanual and bimanual finger responses. Electroencephalogr Clin Neurophysiol 1994; 93: 450-458, doi: 10.1016/01685597(94)90153-8.

37. Klapp ST. Reaction time analysis of two types of motor preparation for speech articulation: action as a sequence of chunks. J Mot Behav 2003; 35: 135-150, doi: 10.1080/0022
2890309602129.

38. Roland PE, Meyer E, Shibasaki T, Yamamoto YL, Thompson CJ. Regional cerebral blood flow changes in cortex and basal ganglia during voluntary movements in normal human volunteers. J Neurophysiol 1982; 48: 467480.

39. Baldauf $D$, Deubel $H$. Visual attention during the preparation of bimanual movements. Vision Res 2008; 48: 549-563, doi: 10.1016/j.visres.2007.11.023.

40. Foltys $H$, Sparing R, Boroojerdi B, Krings T, Meister IG, Mottaghy FM, et al. Motor control in simple bimanual movements: a transcranial magnetic stimulation and reaction time study. Clin Neurophysiol 2001; 112: 265-274, doi: 10.1016/S1388-2457(00)00539-3. 